\title{
Size dependence of the wavefunction of self-assembled InAs quantum dots from time- resolved optical measurements
}

Johansen, Jeppe; Stobbe, Søren; Nikolaev, Ivan S.; Lund-Hansen, Toke; Kristensen, Philip Trøst; Hvam, Jørn Märcher; Vos, Willem L.; Lodahl, Peter

Published in:

Physical Review B Condensed Matter

Link to article, DOI:

10.1103/PhysRevB.77.073303

Publication date:

2008

Document Version

Publisher's PDF, also known as Version of record

Link back to DTU Orbit

Citation (APA):

Johansen, J., Stobbe, S., Nikolaev, I. S., Lund-Hansen, T., Kristensen, P. T., Hvam, J. M., Vos, W. L., \& Lodahl, $P$. (2008). Size dependence of the wavefunction of self-assembled InAs quantum dots from time-resolved optical measurements. Physical Review B Condensed Matter, 77(7), 073303.

https://doi.org/10.1103/PhysRevB.77.073303

\section{General rights}

Copyright and moral rights for the publications made accessible in the public portal are retained by the authors and/or other copyright owners and it is a condition of accessing publications that users recognise and abide by the legal requirements associated with these rights.

- Users may download and print one copy of any publication from the public portal for the purpose of private study or research.

- You may not further distribute the material or use it for any profit-making activity or commercial gain

- You may freely distribute the URL identifying the publication in the public portal 


\title{
Size dependence of the wavefunction of self-assembled InAs quantum dots from time-resolved optical measurements
}

\author{
Jeppe Johansen, ${ }^{1, *}$ Søren Stobbe, ${ }^{1}$ Ivan S. Nikolaev,${ }^{2,3}$ Toke Lund-Hansen, ${ }^{1}$ Philip T. Kristensen, ${ }^{1}$ Jørn M. Hvam, ${ }^{1}$ \\ Willem L. Vos, ${ }^{2,3}$ and Peter Lodahl ${ }^{1, \dagger}$ \\ ${ }^{1}$ COM.DTU, Department of Communications, Optics, and Materials, Technical University of Denmark, DTU-Building 345 V, \\ DK-2800 Kgs. Lyngby, Denmark \\ ${ }^{2}$ Center for Nanophotonics, FOM Institute for Atomic and Molecular Physics (AMOLF), 1098 SJ Amsterdam, The Netherlands \\ ${ }^{3}$ Complex Photonics Systems, MESA ${ }^{+}$Institute for Nanotechnology, University of Twente, 7500 AE Enschede, The Netherlands
}

(Received 10 December 2007; published 12 February 2008)

\begin{abstract}
The radiative and nonradiative decay rates of InAs quantum dots are measured by controlling the local density of optical states near an interface. From time-resolved measurements, we extract the oscillator strength and the quantum efficiency and their dependence on emission energy. From our results and a theoretical model, we determine the striking dependence of the overlap of the electron and hole wavefunctions on the quantum dot size. We conclude that the optical quality is best for large quantum dots, which is important in order to optimally tailor quantum dot emitters for, e.g., quantum electrodynamics experiments.
\end{abstract}

DOI: 10.1103/PhysRevB.77.073303

PACS number(s): 78.67.Hc, 42.50.Ct, 78.47.-p

Semiconductor quantum dots (QDs) have attracted significant attention recently as nanoscale light sources for allsolid-state quantum electrodynamics experiments. ${ }^{1-6}$ Major advancements have culminated in the demonstration of strong coherent coupling between a single QD and the optical mode of a cavity. ${ }^{1-3}$ The coupling strength between the emitter and the cavity is determined by the oscillator strength, which is an intrinsic property of the emitter. For atomic transitions, the oscillator strength attains only discrete values depending on the choice of atom and is determined by the electrostatic potential. In contrast, the QD oscillator strength can be ingeniously tailored due to the influence of size-confinement on the electron-hole wavefunction. ${ }^{7-9}$ Consequently, the oscillator strength can be continuously tuned by varying the size of the QDs. Surprisingly, the exact size dependence of the optical properties of the exciton has remained an open question. Understanding these excitonic optical properties is much required in order to optimally engineer QDs for enhanced light-matter interaction.

In this Brief Report, we present measurements of the oscillator strength of the ground-state exciton in self-assembled QDs. The detailed dependence on the QD size is mapped out by time-resolved measurement of spontaneous emission at different emission energies. We employ the modified local density of optical states (LDOS), caused by reflections in a substrate-air interface, to separate radiative and nonradiative decay contributions. This method was pioneered by Drexhage for dye molecules ${ }^{10}$ and used also to extract the quantum efficiency of erbium ions ${ }^{11}$ and colloidal nanocrystals. ${ }^{12,13}$ Here, we use this method to accurately determine the dependence of the oscillator strength on the quantum dot size. The precise measurements of the oscillator strength allow us to determine the size dependence of the electron and hole wavefunction overlap.

Time-resolved spontaneous emission is measured from a series of samples that contain identical ensembles of InAs QDs positioned at controllable distances to a GaAs-air interface. The wafer is grown by molecular beam epitaxy on a GaAs (100) substrate where 2.0 monolayers of InAs are de- posited at $524{ }^{\circ} \mathrm{C}$ followed by a 30 s growth interruption and deposition of a $300 \mathrm{~nm}$ thick GaAs cap. The QD density is $250 \mu \mathrm{m}^{-2}$. A $50 \mathrm{~nm}$ thick layer of AlAs is deposited $650 \mathrm{~nm}$ below the QDs for an optional epitaxial lift-off. The wafer is processed by standard UV lithography and wet chemical etching, whereby samples with different distances between the QDs and the interface are fabricated on the same wafer [see the inset of Fig. 1(B)]. The distances $z$ from the QD layers to the interface are measured by a combination of secondary ion mass spectroscopy and surface profiling with typical precisions of $\pm 3.0 \mathrm{~nm}$.

The QDs are excited by optical pumping of the wetting layer states at $1.45 \mathrm{eV}$ using $\sim 300 \mathrm{fs}$ pulses from a modelocked Ti:sapphire laser. The excitation spot has a diameter of $\sim 250 \mu \mathrm{m}$ and the excitation density is kept at $7 \mathrm{~W} / \mathrm{cm}^{2}$ with a repetition rate of $82 \mathrm{MHz}$. Under these conditions, less than 0.1 excitons per QD are created, i.e., only light from the QD ground state is observed. The spontaneous emission is collected by a lens (numerical aperture $=0.32$ ), dispersed by a monochromator, and directed onto a silicon avalanche photodiode for time-correlated single-photon counting. ${ }^{14}$ The detection energy is varied between 1.17 and $1.27 \mathrm{eV}$ to probe different subensembles of the inhomogenously broadened ground state. The spectral resolution of the monochromator is $2.6 \mathrm{meV}$, which is narrow relative to the bandwidth of the LDOS changes. The time resolution of the setup is $48 \mathrm{ps}$, given by the full width at half maximum of the total instrument response function. All measurements are performed at $14 \mathrm{~K}$.

Figure 1(A) shows the spontaneous emission decay for QDs positioned at two different distances from the GaAs-air interface and recorded at an emission energy of $1.20 \mathrm{eV}$. A clear change in the decay curve is observed with distance to the interface. The decay of the QD ground state is very well modeled as a biexponential decay, $I(t)=A_{\mathrm{f}} e^{-\Gamma_{\mathrm{f}} t}+A_{\mathrm{s}} e^{-\Gamma_{\mathrm{s}} t}+C$, over the complete time range of the measurement. The background level $C$ is determined by the measured dark count rate and after-pulsing probability of the detector. The fast decay takes place on a time scale of about $1 \mathrm{~ns}$ correspond- 

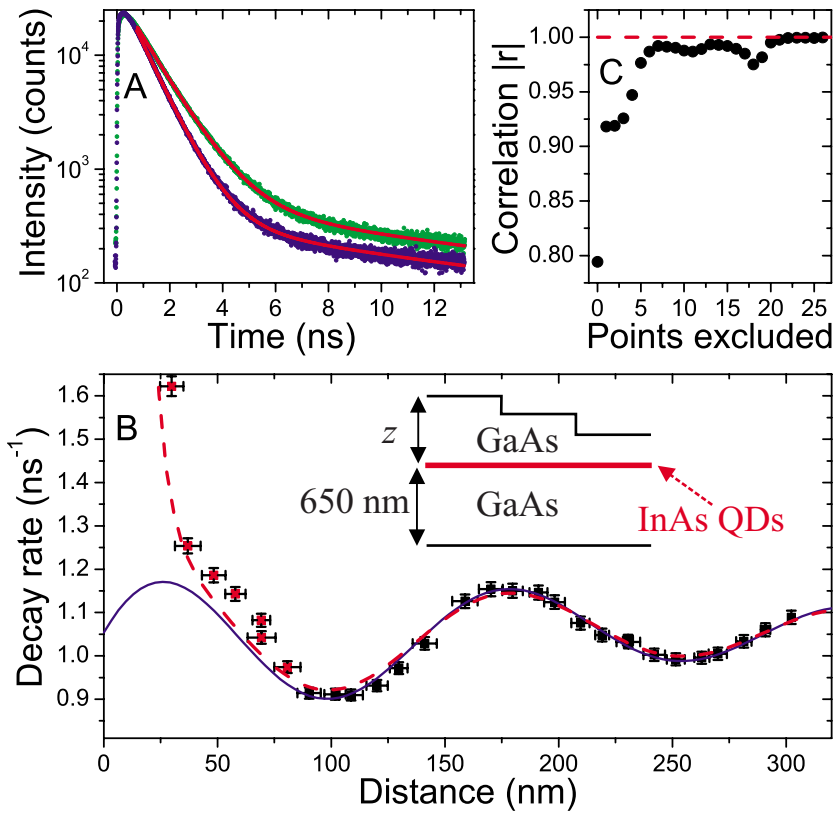

FIG. 1. (Color online) (A) Decay of the spontaneous emission recorded at $1.20 \mathrm{eV}$ for two different distances to the interface of $z=109 \mathrm{~nm}$ (green/light gray, upper curve) and $z=170 \mathrm{~nm}$ (blue/dark gray, lower curve). The solid red/gray lines are biexponential fits resulting in $\Gamma_{\mathrm{f}}=0.91 \mathrm{~ns}^{-1}$ and $\Gamma_{\mathrm{s}}=0.09 \mathrm{~ns}^{-1}$ for $z=109 \mathrm{~nm}$ and $\Gamma_{\mathrm{f}}$ $=1.15 \mathrm{~ns}^{-1}$ and $\Gamma_{\mathrm{s}}=0.10 \mathrm{~ns}^{-1}$ for $z=170 \mathrm{~nm}$. The goodness-of-fit parameters $\chi_{r}^{2}$ are, respectively, 1.17 and 1.11 , close to the ideal value of unity (Ref. 14) verifying the biexponential model. (B) Measured decay rates versus distance $z$ to the GaAs-air interface (squares). Calculated LDOS projected onto a dipole orientation parallel to the interface (solid blue/dark gray line). Calculated LDOS including dissipation at the surface (dashed red/gray line). The inset shows a schematic drawing of the sample. (C) Coefficient of correlation versus the number of data points excluded in the modeling of the data in (B).

ing to the decay of bright excitons in InAs QDs. The slow decay time is approximately $10 \mathrm{~ns}$, does not systematically depend on the distance, and is most likely due to recombination of dark excitons. ${ }^{15}$ In the remainder of this Brief Report, we will focus only on the fast decay rate.

The decay rates measured at $1.20 \mathrm{eV}$ are presented in Fig. 1(B) as a function of distance from the QDs to the interface. A damped oscillation of the total decay rate with distance is observed. The data are compared to the LDOS calculated for GaAs (assuming $n=3.5$ ) and projected onto a dipole orientation parallel to the interface (solid blue/dark gray line). Only the parallel component is relevant since refraction in the interface reduces the solid angle for light collection, and additionally, the QD orientation is predominantly parallel to the interface. ${ }^{16}$ The measured decay rate $\Gamma(\omega, z)$ is the sum of nonradiative $\Gamma_{\text {nrad }}(\omega)$ and radiative $\Gamma_{\text {rad }}(\omega, z)$ decay rates. The latter is proportional to the projected LDOS $\rho(\omega, z)$ and depends explicitly on the distance $z$ to the interface and the optical frequency $\omega$. It is calculated as the sum over all available electromagnetic modes projected onto the orientation of the dipole, and it is obtained using a Greens function approach. ${ }^{17}$ We define the radiative decay rate for QDs in a homogeneous medium $\Gamma_{\mathrm{rad}}^{\mathrm{hom}}(\omega)$ and express the measured total decay rate as

$$
\Gamma(\omega, z)=\Gamma_{\mathrm{nrad}}(\omega)+\Gamma_{\mathrm{rad}}^{\mathrm{hom}}(\omega) \frac{\rho(\omega, z)}{\rho_{\mathrm{hom}}(\omega)},
$$

where $\rho_{\text {hom }}(\omega)$ is the LDOS of a homogeneous medium of GaAs.

Excellent agreement between experiment and theory is observed in Fig. 1(B) for distances $z \geqslant 75 \mathrm{~nm}$. This explicitly confirms the validity of the theoretical model used to extract properties of the emitter as opposed to a previous work. ${ }^{12}$ For QDs closer than $75 \mathrm{~nm}$ to the GaAs-air interface, the measured decay rates are systematically larger than the calculated rates. We exclude that this effect is due to tunneling out of the QDs, which has been observed only within $15 \mathrm{~nm}$ from a surface. ${ }^{18}$ An increased nonradiative loss may be due to scattering or absorption at the surface of the etched samples. This dissipation at the surface is modeled as a thin absorbing surface layer, which creates an optical surface state. The dashed line in Fig. 1(B) is obtained by including a $5 \mathrm{~nm}$ thick layer with refractive index of $3.5+1.0 i$, which leads to increased rates near the interface in agreement with the experimental data.

For QDs sufficiently far away from the interface, the influence of any surface effects is negligible, and our data can be used to reliably extract QD properties. We determine the data points that are not influenced by the dissipation at the surface as follows: Eq. (1) reveals a linear relation between the measured rate and the calculated normalized LDOS. We therefore perform a linear regression analysis and obtain the linear correlation parameter $|r|$ as the data close to the interface are excluded point by point [cf. Fig. 1(C)]. After excluding the seven closest data points, the correlation parameter converges to unity, hence Eq. (1) is valid. By comparing experiment and theory, we determine the radiative and nonradiative decay rates at $1.20 \mathrm{eV}$ to be $\Gamma_{\mathrm{rad}}^{\mathrm{hom}}$ $=0.95 \pm 0.03 \mathrm{~ns}^{-1}$ and $\Gamma_{\text {nrad }}=0.11 \pm 0.03 \mathrm{~ns}^{-1}$, which are the most accurate results to date for QDs.

The measurements have been performed for six different energies within the inhomogenously broadened emission spectrum of the QDs. The inhomogeneous broadening reflects the different sizes of QDs such that small QDs correspond to high emission energies and vice versa. The radiative and nonradiative decay rates extracted from the measurements are plotted in Fig. 2. The increased nonradiative recombination rate at higher energies could indicate that carriers can be trapped at the QD surface since the relative importance of the surface is large for small QDs. While such a size dependence would be general for all QDs, the absolute values of the nonradiative rates could depend on sample growth. Surprisingly, the radiative rate is found to decrease with increasing energy. This behavior is due to the decrease of the overlap between the electron and hole wavefunctions as the size of the QD is reduced, as discussed below. Our method allows us to extract the size dependence of the QD emission without any implicit assumption of vanishing nonradiative recombination, as opposed to previous works. ${ }^{8,19}$ In fact, such an assumption would, in our case, lead to the incorrect conclusion of an increased wavefunction overlap with reduced QD size. 


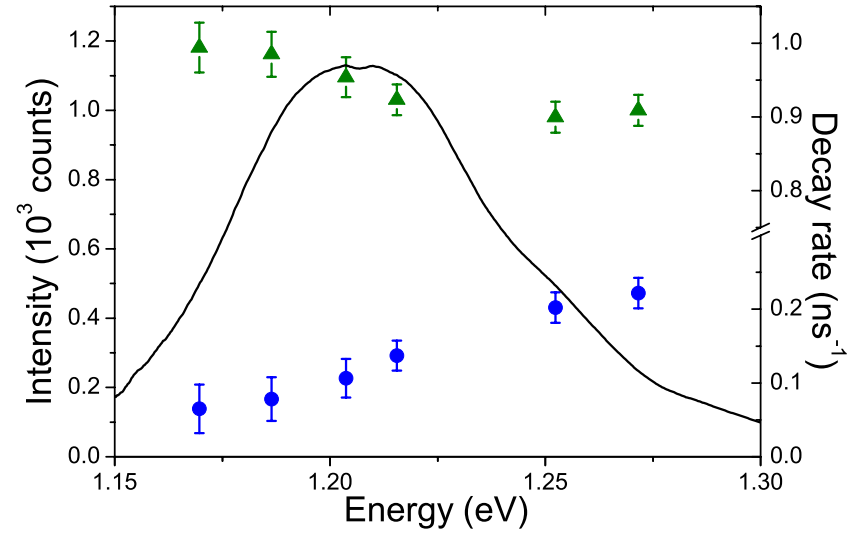

FIG. 2. (Color online) Left axis: photoluminescence from the inhomogenously broadened ground state measured at $z=281 \mathrm{~nm}$ (solid line). Right axis: radiative (green/light gray triangles) and nonradiative (blue/dark gray circles) decay rates versus emission energy.

Fermi's golden rule relates the radiative decay rate and the oscillator strength,

$$
f_{\mathrm{osc}}(\omega)=\frac{6 m_{e} \epsilon_{0} \pi c_{0}^{3}}{q^{2} n \omega^{2}} \Gamma_{\mathrm{rad}}^{\mathrm{hom}}(\omega),
$$

where $n$ is the refractive index of GaAs, $\omega$ is the frequency of the optical transition, $m_{e}$ is the electron mass, $\epsilon_{0}$ is the vacuum permittivity, $q$ is the elementary charge, and $c_{0}$ is the speed of light in vacuum. For the QDs emitting at $\hbar \omega$ $=1.20 \mathrm{eV}$, the measured value of $\Gamma_{\mathrm{rad}}^{\mathrm{hom}}$ results in an oscillator strength of $f_{\mathrm{osc}}=13.0 \pm 0.4$. For comparison, various estimates of the oscillator strength based on absorption measurements have been reported in the literature and are generally in the range of $f_{\text {osc }}=5-10 .{ }^{20,21}$ However, the technique implemented here provides unprecedented precision since it only relies on accurate measurements of the distance of the QDs to the interface and is independent of, e.g., the QD density. Additionally, the quantum efficiency, i.e., the ratio of the radiative decay rate to the total decay rate, is extracted. We find $Q E=90 \% \pm 4 \%$ at the emission energy of $1.20 \mathrm{eV}$, which confirms that a high quantum efficiency is feasible with an ensemble of emitters and not only with single QDs. ${ }^{12}$ The intrinsically high QD quantum efficiency can be further increased by tuning the size as discussed below.

The energy dependence of the oscillator strength and the quantum efficiency are presented in Fig. 3(A). Both quantities are seen to decrease with increasing energy. The quantum efficiency decreases from around $95 \%$ to $80 \%$ and the oscillator strength from 14.5 to 11 over the inhomogenously broadened emission spectrum. This result shows that large QDs with a high exciton confinement potential have much better optical properties than smaller QDs. Our results thus shed light on the optimum design of solid-state QED experiments, and strong coupling was indeed observed for large QDs. ${ }^{1-3}$

Our measurements provide insight into the size dependence of the QD wavefunctions. The spatial overlap between the electron and hole wavefunctions $\left|\Psi_{e, h}(\omega)\right\rangle$ can be ob-

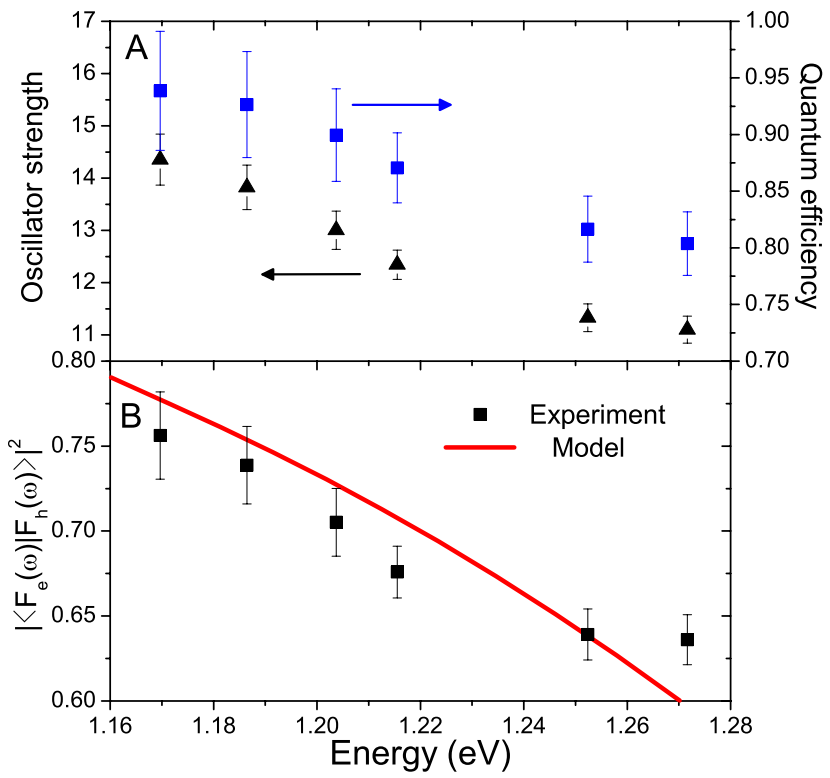

FIG. 3. (Color online) (A) Oscillator strength (triangles) and quantum efficiency (squares) versus energy (B) Measured (squares) and calculated (red/gray curve) energy dependence of the overlap of the envelope wavefunctions of electrons and holes.

tained from the oscillator strength. Within the effective-mass approximation, valid in the strong confinement limit when Coulomb effects are negligible, the electron (or hole) wavefunction can be factorized in a conduction (or valence) band Bloch wavefunction for InAs $\left|u_{c / v}\right\rangle$ and an electron (or hole) envelope function $\left|F_{e / h}(\omega)\right\rangle$. The overlap of the electron and hole wavefunctions is related to the oscillator strength via $^{19,22,23}$

$$
\left|\left\langle F_{e}(\omega) \mid F_{h}(\omega)\right\rangle\right|^{2}=\frac{m_{e} \hbar \omega}{6\left|\left\langle u_{v}|\hat{\mathbf{e}} \cdot \mathbf{p}| u_{c}\right\rangle\right|^{2}} \times f_{\text {osc }}(\omega),
$$

where $\hat{\mathbf{e}}$ is the polarization unit vector of the electromagnetic field and $\mathbf{p}$ is the electron momentum. Evaluation of the matrix element is performed as an average over all possible orientations of the polarization vector. As strain lifts the degeneracy of the light-hole and heavy-hole bands, only transitions from the conduction band to the heavy-hole band are included. The result can be expressed as $\left|\left\langle u_{v}|\hat{\mathbf{e}} \cdot \mathbf{p}| u_{c}\right\rangle\right|^{2}$ $=m_{e} E_{p} / 6$, where $E_{p}=22.2 \mathrm{eV}$ is the Kane energy for bulk InAs. ${ }^{23}$ From Eq. (3) and the measured radiative decay rates, the wavefunction overlap is obtained [see Fig. 3(B)]. The wavefunction overlap decreases from about 0.75 at $1.17 \mathrm{eV}$ to 0.63 at $1.27 \mathrm{eV}$. The reduction in the oscillator strength stems from the increased mismatch between the electron and hole wavefunctions with decreasing QD size. The reduction of the wavefunction overlap is due to the more sensitive size dependence of the electron wavefunction compared to the hole wavefunction due to their difference in effective mass. As the size of the QD is decreased, the electron will penetrate deeper into the barrier than the hole, thus reducing the overlap. ${ }^{24}$ The detailed understanding of how the size affects the overlap between the electron and hole wavefunctions is 
crucial in order to optimally tailor QDs for efficient coupling to light.

The observed energy dependence of the oscillator strength is compared to a simple effective-mass QD model. We use finite-element-method calculations to obtain the energy levels and the corresponding wavefunctions of the electron and hole. The curve in Fig. 3(B) displays the wavefunction overlap calculated for a lens-shaped QD with a radius of $7 \mathrm{~nm}$ and a height varying between 1.8 and $3.0 \mathrm{~nm}$. The following realistic parameters are used: a wetting layer thickness of $0.3 \mathrm{~nm}, 60 \%$ of the band-edge discontinuity is in the conduction band, and the GaAs content in the QDs is taken to be $25 \%$. Good agreement with the experimental data is observed, and the theory clearly confirms a pronounced reduction of the electron-hole wavefunction overlap as the size of the QD is decreased. The general validity has been tested by calculating for different sizes, shapes, and amount of GaAs content in the QD, and they all show a decrease of overlap with increasing energy. The same behavior is also obtained from more involved QD models also in the presence of strain. ${ }^{25}$ Interestingly, the radiative rate was observed to increase with energy for colloidal nanocrystals in agreement with theory, ${ }^{19}$ which is opposite to the results reported here for InAs QDs. This illustrates a striking difference in the optical properties of colloidal nanocrystals compared to selfassembled QDs, which is due to their different sizes and confinement potentials.
In summary, we have measured the radiative and nonradiative decay rates of InAs QDs by employing the modified LDOS near a dielectric interface. The oscillator strength and quantum efficiency of the QDs and their dependence on the emission energy were accurately determined. The radiative decay rate decreases with increasing energy, leading to a reduction of the oscillator strength. In contrast, the nonradiative recombination rate increases with increasing energy corresponding to a reduction of the quantum efficiency. Consequently, QDs emitting on the low-energy side of the inhomogenously broadened ground-state transition are most suitable as nanophotonic light sources due to their optimized optical properties. The experimental findings are explained by a model of the QD taking the size dependence of the wavefunctions into account. Our results demonstrate how QD wavefunctions can be tailored to achieve improved coupling to light, which is needed in order to take full advantage of the potential of quantum electrodynamics devices based on QDs.

We thank Martin Olesen, Torben Kristensen and Stig Salomonsen for supplying the program code for the QD model and Mads Lykke Andersen and Ad Lagendijk for stimulating discussions. We gratefully acknowledge the Danish Research Agency (division FNU) for financial support. This work was part of the project "Qphoton." I.S.N. and W.L.V. are supported by FOM and NWO-Vici. *jjo@com.dtu.dk

†pel@com.dtu.dk

${ }^{1}$ J. P. Reithmaier, G. Sȩk, A. Löffler, C. Hofmann, S. Kuhn, S. Reitzenstein, L. V. Keldysh, V. D. Kulakovskii, T. L. Reinecke, and A. Forchel, Nature (London) 432, 197 (2004).

${ }^{2}$ T. Yoshie, A. Scherer, J. Hendrickson, G. Khitrova, H. M. Gibbs, G. Rupper, C. Ell, O. B. Shchekin, and D. G. Deppe, Nature (London) 432, 200 (2004).

${ }^{3}$ E. Peter, P. Senellart, D. Martrou, A. Lemaître, J. Hours, J. M. Gérard, and J. Bloch, Phys. Rev. Lett. 95, 067401 (2005).

${ }^{4}$ P. Lodahl, A. F. van Driel, I. S. Nikolaev, A. Irman, K. Overgaag, D. Vanmaekelbergh, and W. L. Vos, Nature (London) 430, 654 (2004).

${ }^{5}$ A. Kress, F. Hofbauer, N. Reinelt, M. Kaniber, H. J. Krenner, R. Meyer, G. Böhm, and J. J. Finley, Phys. Rev. B 71, 241304(R) (2005).

${ }^{6}$ D. Englund, D. Fattal, E. Waks, G. Solomon, B. Zhang, T. Nakaoka, Y. Arakawa, Y. Yamamoto, and J. Vučković, Phys. Rev. Lett. 95, 013904 (2005).

${ }^{7}$ L. C. Andreani, G. Panzarini, and J. M. Gérard, Phys. Rev. B 60, 13276(R) (1999).

${ }^{8}$ J. Hours, P. Senellart, E. Peter, A. Cavanna, and J. Bloch, Phys. Rev. B 71, 161306(R) (2005).

${ }^{9}$ J. J. Finley, M. Sabathil, P. Vogl, G. Abstreiter, R. Oulton, A. I. Tartakovskii, D. J. Mowbray, M. S. Skolnick, S. L. Liew, A. G. Cullis et al., Phys. Rev. B 70, 201308(R) (2004).

${ }^{10}$ K. H. Drexhage, J. Lumin. 1-2, 693 (1970).

${ }^{11}$ E. Snoeks, A. Lagendijk, and A. Polman, Phys. Rev. Lett. 74, 2459 (1995).

${ }^{12}$ X. Brokmann, L. Coolen, M. Dahan, and J. P. Hermier, Phys.
Rev. Lett. 93, 107403 (2004).

${ }^{13}$ R. J. Walters, J. Kalkman, A. Polman, H. A. Atwater, and M. J. A. de Dood, Phys. Rev. B 73, 132302 (2006).

${ }^{14} \mathrm{~J}$. R. Lakowicz, Principles of Fluorescence Spectroscopy (Springer, New York, 2006).

${ }^{15}$ I. Favero, G. Cassabois, C. Voisin, C. Delalande, P. Roussignol, R. Ferreira, C. Couteau, J. P. Poizat, and J. M. Gérard, Phys. Rev. B 71, 233304 (2005).

${ }^{16}$ S. Cortez, O. Krebs, P. Voisin, and J. M. Gérard, Phys. Rev. B 63, 233306 (2001).

${ }^{17}$ R. R. Chance, A. H. Miller, A. Prock, and R. Silbey, J. Chem. Phys. 63, 1589 (1975).

${ }^{18}$ C. F. Wang, A. Badolato, I. Wilson-Rae, P. M. Petroff, E. Hu, J. Urayama, and A. Imamoglu, Appl. Phys. Lett. 85, 3423 (2004).

${ }^{19}$ A. F. van Driel, G. Allan, C. Delerue, P. Lodahl, W. L. Vos, and D. Vanmaekelbergh, Phys. Rev. Lett. 95, 236804 (2005).

${ }^{20}$ D. Birkedal, J. Bloch, J. Shah, L. N. Pfeiffer, and K. West, Appl. Phys. Lett. 77, 2201 (2000).

${ }^{21}$ R. J. Warburton, C. S. Dürr, K. Karrai, J. P. Kotthaus, G. Medeiros-Ribeiro, and P. M. Petroff, Phys. Rev. Lett. 79, 5282 (1997).

${ }^{22}$ C. Delerue and M. Lannoo, Nanostructures-Theory and Modeling (Springer-Verlag, Berlin, 2004).

${ }^{23}$ J. Singh, Semiconductor Optoelectronics (McGraw-Hill, New York, 1995).

${ }^{24}$ G. A. Narvaez, G. Bester, and A. Zunger, J. Appl. Phys. 98, 043708 (2005).

${ }^{25}$ A. D. Andreev and E. P. O'Reilly, Appl. Phys. Lett. 87, 213106 (2005). 